\title{
Cell-Specific Activity of the Modulator Region in the Human Cytomegalovirus Major Immediate-Early Gene $\dagger$
}

\author{
HENRYK LUBON,${ }^{1}$ PETER GHAZAL,${ }^{2}$ LOTHAR HENNIGHAUSEN,${ }^{1 *}$ CATHERINE REYNOLDS-KOHLER, ${ }^{2}$ \\ CURTIS LOCKSHIN, ${ }^{2}$ AND JAY NELSON ${ }^{2}$
}

\begin{abstract}
Laboratory of Biochemistry and Metabolism, National Institute of Diabetes and Digestive and Kidney Diseases, Building 10, Room 9N113, Bethesda, Maryland 20892, ${ }^{1}$ and Department of Immunology, Scripps Clinic and Research Foundation, La Jolla, California $92037^{2}$
\end{abstract}

Received 7 June 1988/Accepted 9 November 1988

\begin{abstract}
In this paper we demonstrate that modulator sequences upstream of the enhancer of the major immediateearly promoter of human cytomegalovirus exert a differential effect on the level of transcription in a variety of cells and that this region has the capacity to interact with specific nuclear protein. Depending on the cell type, these modulator sequences increased or decreased transcriptional activation from the IE1 gene promoterenhancer. The cell lines identified in this report should be useful to study the molecular mechanism of cell-specific transcriptional repression and activation exerted by the major immediate-early promoter upstream region.
\end{abstract}

Although human cytomegalovirus (HCMV) nonproductively infects cells of many types from humans $(8,16,17)$ and other species $(2,11)$, the virus replicates in vitro only in fibroblasts of human origin. Transcription of the first viral genes expressed, the immediate-early (IE) genes, are mainly dependent on host cell factors $(1,5-7,20,21)$, suggesting that this may constitute the primary event at which expression of the viral genome is regulated. Regulatory sequences of the HCMV major IE gene, the IE1 gene, encompass a polymerase II promoter between +1 and $-65(6,19)$; a strong and complex enhancer between -66 and $-524(1,5$, 7), which is active in many different cell types; a cluster of nuclear factor 1-binding sites between -600 and $-720(9$, 10); and modulator sequences between -750 and -1145 (14, 15). These modulator sequences negatively regulate expression in nonpermissive undifferentiated teratocarcinoma (Tera-2) cells but positively influence expression in permissive differentiated cells $(14,15)$. In the present study we have addressed the question of whether the modulator region is functional in cell types other than the embryonal carcinoma cell system and whether indeed it may contribute to cellspecific expression.

Recombinant plasmids containing the modulator $(\Delta 1145)$ or with the modulator sequences truncated $(\Delta 525)$, which were previously used to define the modulator region (15), were analyzed for transcriptional activity by transient-transfection experiments in a variety of cells. Monolayer cells (SW480 and 293) were transfected by the calcium phosphate technique (15), and nonadherent cells (H9, Jurkat, Namalwa, Raji, and U937) were transfected by the DEAE-dextran chloroquine technique. Approximately $10^{7}$ cells were cotransfected with $30 \mu \mathrm{g}$ of the respective plasmid and $5 \mu \mathrm{g}$ of the transfected control plasmid pRSVL, a plasmid containing the luciferase gene under the control of the Rous sarcoma virus long terminal repeat (LTR) (3). Cells were harvested after $48 \mathrm{~h}$, and protein reporter functions were assayed as described previously (15). The relative chloramphenicol acetyltransferase (CAT) activities directed by plasmids

* Corresponding author.

$\dagger$ Publication no. 5600-IMM from the Department of Immunology, Scripps Clinic and Research Foundation.
$\Delta 1145$ and $\Delta 525$ in cells of various lineages were measured by scintillation counting of conversion rates from chloramphenicol to its acetylated forms. All cells were cotransfected with pRSV-luciferase as a transfection control (3), and the results with $\Delta 1145$ and $\Delta 525$ were adjusted according to the relative luciferase activity in the cell extracts. The effect of the modulator sequences on CAT activity differed depending on cell type (Fig. 1). A twofold negative effect was observed in H9, U937, and SW480 cells, but expression in Jurkat, 293, Raji, and Namalwa cells was barely affected. In B cells the expression was augmented about three- to fourfold by the upstream sequences. These results clearly indicate that the HCMV modulator sequences can influence IE promoterenhancer activity in vivo in cell types other than teratocarcinoma cells. In particular, the cis-acting sequences negatively modulate expression in some $\mathrm{T}$ cells and rectal epithelial cells, in which HCMV is found during natural infection.

To identify functional properties of this region in vitro, we analyzed transcriptional activities of IE1 promoter-enhancer templates, with or without the upstream sequences, in nuclear extracts from a variety of cell lines. Nuclear extracts were prepared from nuclei $\left(2 \times 10^{9}\right.$ to $\left.5 \times 10^{9}\right)$ of Jurkat, CEM, H9, U937, Raji, Epstein-Barr virus-transformed B cells, T47-D, HeLa S3, and 293 cells essentially as described previously (4). Figure $2 \mathrm{~A}$ shows runoff transcription products from templates $\Delta 1145$ and $\Delta 524$ with nuclear extracts from the various cell types. Conditions for in vitro transcription were as previously described (5), except that a $30-\mu 1$ reaction volume was used which included $300 \mu \mathrm{M}$ CTP, GTP, and ATP and $70 \mu \mathrm{g}$ of nuclear protein. Although the transcriptional efficiencies in nuclear extracts from each cell line varied among preparations, the relative number of transcriptions of $\Delta 1145$ to $\Delta 524$ in each cell line were similar. Greatly reduced transcriptional activity from $\Delta 1145$ was observed in nuclear extracts from CEM, U937, and Raji cells. Slightly lower levels of repression were detected in HeLa S3 and H9 cells, whereas extracts from Jurkat, 293, T47-D, and B cells exerted only a marginal effect.

To assess the extent of specificity of the cell-specific transcriptional modulation for the HCMV IE1 promoter, we performed control experiments and analyzed transcription 


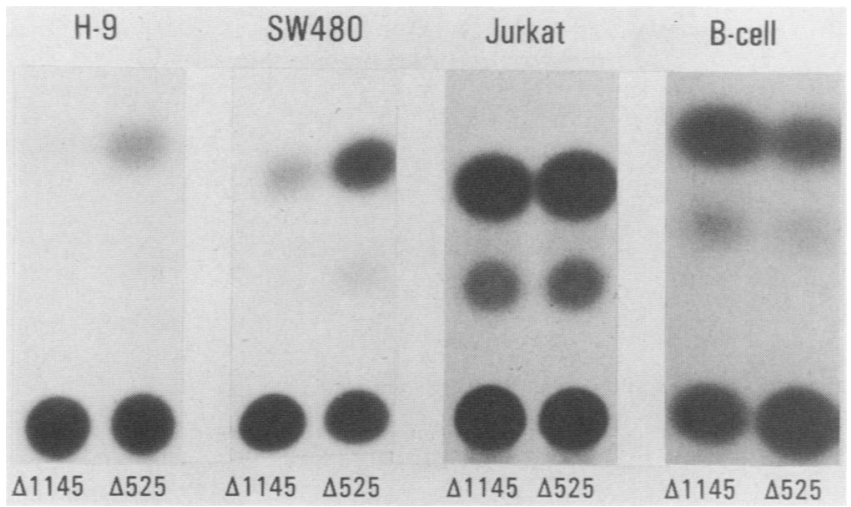

FIG. 1. Representative CAT assays demonstrating the effect of the modulator sequence of HCMV IE promoter-enhancer activity. H9, SW480, Jurkat, and B cells were transfected with plasmids containing the CAT gene under the control of the HCMV IE promoter-enhancer with $(\Delta 1145)$ and without $(\Delta 525)$ the modulator sequences. We tested cell extracts $(100 \mu \mathrm{g})$ for CAT activity by resolving chloramphenicol from the acetylated forms by thin-layer chromatography. All cells were cotransfected with pRSV-luciferase as a transfection control (3), and extracts were assayed for activity. The autoradiograms are representative samples of experiments with these different cell lines.

from two human immunodeficiency virus (HIV) LTR templates. The HIV LTR has been shown in vivo (18) and in vitro (13) to contain a negative transcriptional-regulatory element upstream of -278 . In nuclear extracts from CEM, Raji, T47-D, and B cells, the relative transcriptional activities of HIV templates with deletion endpoints at -453 and -278 (Fig. 3B) were significantly different from those obtained with the two IE1 templates (Fig. 2B). This suggests that the negative regulatory element in the HIV LTR is different from that in the IE1 gene. Similar to the IE1 template $\Delta 1145$, the HIV template $\Delta 453$ showed transcriptional inhibition in $\mathrm{H} 9$ and $\mathrm{U} 937$ extracts. In general, the observed in vivo modulatory effect correlated well with the in vitro experiments. However, it should be noted that the in vitro experiments involve the use of naked DNA templates with a subfraction of soluble nuclear factors. Also, they do no exclude the possibility that other sequences have additional effects. For example, $\Delta 524$ but not $\Delta 1145$ contains 50-base-pair IE1 RNA-encoding sequences which may stimulate IE1 gene expression in tissue culture cells. Therefore, such assays may only be a partial reflection of the functional aspects of a specific region.

Since the activities of the modulator region may be mediated by the binding of transcription factors, we have further investigated the ability of this region to interact with nuclear proteins. Initially, HeLa S3 cells nuclear extract was chosen because of its ability to repress in vitro transcription from templates containing the modulator region. In an electrophoretic mobility shift analysis, the Pst $\mathrm{I}-$ AluI fragment from -1145 to -773 and nuclear proteins formed one major and several minor complexes ( Fig. $4 \mathrm{~A}$, lane 2), suggesting the binding of several factors. Competition experiments established the sequence specificity of these complexes. The predominant complex $c$ was inhibited by an excess of the unlabeled PstI-AluI fragment (Fig. 4A, lane 5) but not by a fragment from the CAT gene (lane 4) or by restriction fragments from pUC19 (lane 3). Complex $a$ was inhibited by the unlabeled probe and by pUC19 fragments but not by the CAT gene fragment (Fig. 4A). To determine whether the nucleoprotein complexes were specific to the modulator

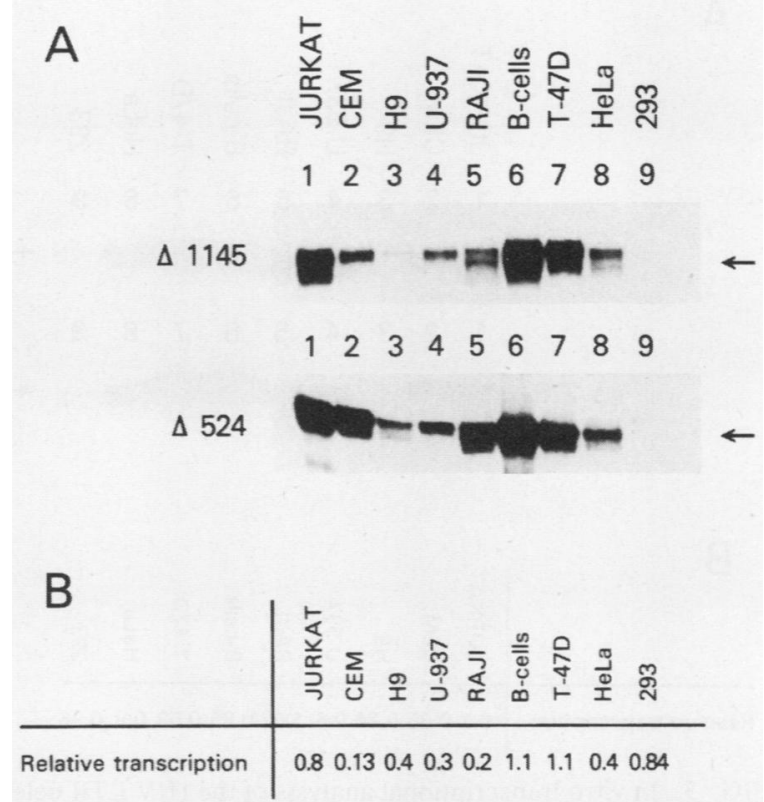

FIG. 2. In vitro transcriptional activity of $\Delta 1145$ and $\Delta 524$. (A) Runoff transcripts originating at the in vitro start site of the respective templates are indicated by arrows. The cell lines used to prepare the nuclear extracts are shown above the correspondingly numbered lanes. HCMV IE1 gene templates were prepared by cutting the plasmids $\Delta 1145(15)$ and $\Delta 524$ (5) with $P v u I I$ and $H i n d I I I$. HCMV sequences in $\Delta 1145$ are from -1145 to +7 , and those in $\Delta 524$ are from -524 to +55 . Runoff transcripts originating at the in vivo start site of the respective templates are indicated by arrows. (B) Relative transcription of $\Delta 1145$ to $\Delta 524$. Quantitation of transcription was achieved by densitometer scanning (model 620 video densitometer; Bio-Rad Laboratories) of several autoradiograms corresponding to different exposure times.

region of the IE1 gene, competition assays were performed with two fragments from the IE1 gene enhancer and promoter region (5). A 50-fold molar excess of an enhancer fragment did not compete for binding, whereas the presence of an enhancer-promoter fragment specifically reduced complex $c$, but not complex $a$ (Fig. 4A, lanes 6 and 7). It is possible that the proteins forming complex $c$ recognize similar sequence structures in the promoter and upstream regions or that such factor(s) recognize more than one sequence. On the basis of results from binding assays with smaller and overlapping restriction fragments, sequences between -970 and -912 participate in the formation of complex $c$ (data not shown).

To determine whether nucleoprotein complexes were specific to HeLa cell nuclear proteins, we analyzed binding to the modulatory region by using the library of transcriptionally active nuclear extracts. Nuclear proteins from $\mathrm{T}$ cells (Jurkat, CEM, and H9), monocytes (U937), Burkitt lymphoma cells (Raji), Epstein-Barr virus-immortalized B cells, mammary epithelial cells (T47-D), and embryonic kidney cells (293) induced the formation of complexes (Fig. 4B) with similar mobilities to those found in the HeLa cell nuclear extract. An additional complex with a mobility greater than that of complex $a$, but less than that of complex $b$, was observed predominantly in the nuclear extract from T47-D cells and to a lesser extent in extracts from Jurkat and U937 cells (Fig. 4B, lanes 8, 3, and 5, respectively). Complexes $a$, $b$, and $c$ were generated by nuclear factors with similar 


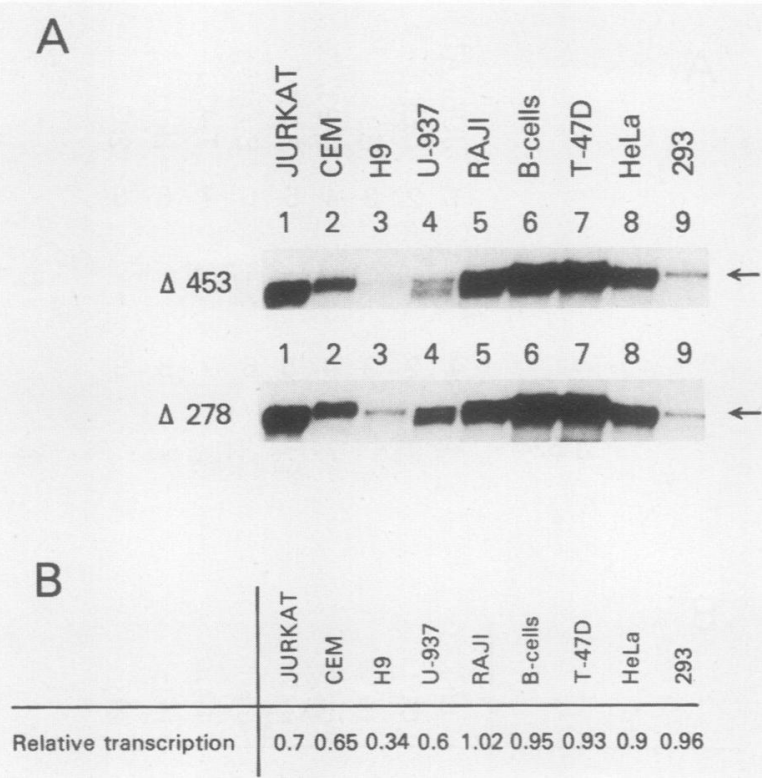

FIG. 3. In vitro transcriptional analysis of the HIV LTR deletion mutants -453 and -278 . (A) Runoff transcripts originating at the in vivo start site of the respective templates are indicated by arrows. (B) Relative transcription of -453 and -278 . For a description, see the legend to Fig. 2. HIV templates were prepared by cutting plasmids containing the HIV LTR 5 ' deletion mutants $453 / 80$ and $-278 / 80$ ligated to the CAT (18) gene with EcoRI. The resulting runoff transcripts were about 410 base pairs $(13,18)$.

efficiencies in each tested extract, suggesting that this modulator region is a target for related DNA-binding proteins which are present in all cell types.

Proteins from each of the transcriptionally active nuclear extracts formed generally similar patterns of protein-DNA complexes with the modulator region in the mobility shift assay; we did not observe a simple correlation between the transcriptional activities and protein binding. This could reflect the inability of the mobility shift assay to resolve complexes containing similar and/or posttranslationally modified proteins. Alternatively, the activity of this region could be dependent on transcription factors acting through protein-protein interactions and may not necessarily be detected in this assay. We also cannot exclude the possibility that enhancer and promoter sequences absent in the binding assay are required for the stability of a complex associated with the modulator region. It has been suggested that nuclear factor 1-binding sites between -775 and -665 might be involved in the modulator activities $(10,12,14,15)$. However, the capacity of nuclear proteins to interact with these binding sites did not vary significantly among extracts from the different cell lines (data not shown). Perhaps the simplest explanation would account for the fact that the IE regulatory sequences interface with a wide variety of transcription factors (5-7), and, as a consequence, it is the combinatorial interactions between those positive and negative elements within any given cell that ultimately determine the extent of transcription.

Finally, in this report we have shown that the modulator region of the HCMV IE1 gene modulates the level of transcription in a cell-specific manner. Indeed, the differential effect exerted by the modulator region in a variety of cell types may be an important determinant of viral latency and/or reactivation. By studying a battery of differentially

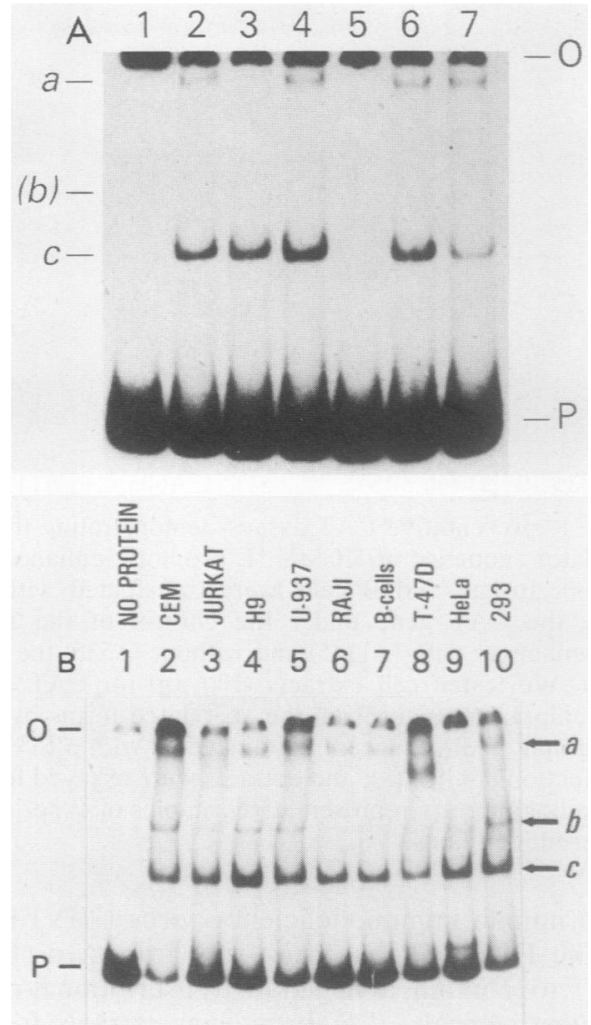

FIG. 4. Binding of nuclear proteins to the PstI-AluI (-1145 to -773) fragment of the HCMV IE1 gene. One nanogram of endlabeled fragment was incubated with $2 \mu \mathrm{g}$ of poly(dI-dC) and $1 \mu \mathrm{g}$ of nuclear extract. Binding conditions and electrophoresis were as described previously (6). (A) Mobility shift assay with HeLa cell nuclear extract and a 50 -fold molar excess of different competitor DNAs. Lane 1, probe alone; lanes 2 to 7 , probe with nuclear extract and the following competitor DNAs: poly(dI-dC) (lane 2), pUC19 cut with HaeIII (lane 3), a 250-base-pair EcoRI-HindIII fragment from the CAT gene (lane 4), unlabeled PstI-AluI fragment (lane 5), enhancer fragment spanning nucleotides -524 to -311 (lane 6), enhancer-promoter fragment spanning nucleotides -185 to -14 (lane 7). (B) Protein-DNA complexes formed between the PstI-AluI fragment and nuclear extracts from different cell lines. Lane 1, probe; lanes 2 to 10 , binding with nuclear extracts from CEM cells (lane 2), Jurkat cells (lane 3), H9 cells (lane 4), U937 cells (lane 5), Raji cells (lane 6), B cells (lane 7), T47-D cells (lane 8), HeLa S3 cells (lane 9), and 293 cells (lane 10). $O$ and $P$ indicate the origin of the gel and unbound probe, respectively.

active nuclear extracts now at our disposal, it should be possible to investigate the molecular basis of the influence of the modulator region on transcription and thus gain a better understanding of the functioning of the viral nucleic acids in a cell.

The HIV LTR deletion plasmids described in reference 18 were generated by S. Josephs and F. Wong-Staal and kindly provided by R. Robbins.

This work was supported in part by Public Health Service grant AI-21640 from the National Institutes of Health, a Faculty Research Award from the American Cancer Society (to J.N.), and by "Funds for AIDS Targeted Antiviral Agents" through the Office of the Director of the National Institutes of Health (to L.H.).

\section{LITERATURE CITED}

1. Boshart, M., F. Weber, G. Jahn, K. Dorsch-Hasler, B. Fleckenstein, and W. Schaffner. 1985. A very strong enhancer is located 
upstream of an immediate-early gene of human cytomegalovirus. Cell 41:521-530.

2. DeMarchi, J. M. 1983. Post-transcriptional control of human cytomegalovirus gene expression. Virology 124:390-402.

3. DeWet, J. R., K. V. Wood, M. DeLuca, D. R. Helinski, and S. Subramani. 1987. Firefly luciferase gene: structure and expression in mammalian cells. Mol. Cell. Biol. 7:725-735.

4. Dignam, J. D., R. M. Lebowitz, and R. G. Roeder. 1983. Accurate transcription initiation by RNA polymerase II in a soluble extract from isolated mammalian nuclei. Nucleic Acids Res. 11:1475-1489.

5. Ghazal, P., H. Lubon, B. Fleckenstein, and L. Hennighausen. 1987. Binding of transcription factors and creation of a large nucleoprotein complex on the human cytomegalovirus enhancer. Proc. Natl. Acad. Sci. USA 84:3658-3662.

6. Ghazal, P., H. Lubon, and L. Hennighausen. 1988. Specific interactions between transcription factors and the promoterregulatory region of the human cytomegalovirus major immediate-early gene. J. Virol. 62:1076-1079.

7. Ghazal, P., H. Lubon, and L. Hennighausen. 1988. Multiple sequence-specific transcription factors modulate cytomegalovirus activity in vitro. Mol. Cell. Biol. 8:1809-1811.

8. Gonczol, E., P. W. Andrews, and S. A. Plotkin. 1984. Cytomegalovirus replicates in differentiated but not in undifferentiated human embryonal carcinoma cells. Science 224:159-161.

9. Hennighausen, L., and B. Fleckenstein. 1986. Nuclear factor 1 interacts with five DNA elements in the promoter region of the human cytomegalovirus major immediate-early gene. EMBO J. 5:1367-1371.

10. Jeang, K., D. R. Rawlins, P. J. Rosenfeld, J. H. Shero, T. J. Kelly, and G. S. Hayward. 1987. Multiple tandemly repeated binding sites for cellular nuclear factor 1 that surround that major immediate-early promoters of simian and human cytomegalovirus. J. Virol. 61:1559-1570.

11. LeFemina, R. L. 1983. Replicative forms of human cytomegalovirus DNA with joined termini are found in permissively infected human cells but not in non-permissive Balb/c-3T3 mouse cells. J. Gen. Virol. 64:373-389.
12. LaFemina, R., and G. S. Hayward. 1986. Constitutive and retinoic acid-inducible expression of cytomegalovirus immediate-early genes in human teratocarcinoma cells. J. Virol. 58: 434-440.

13. Lubon, H., P. Ghazal, J. A. Nelson, and L. Hennighausen. 1988. Cell-specific activity of the human immunodeficiency virus enhancer repeat in vitro. AIDS Res. Human Retroviruses 4: 381-391.

14. Nelson, J. A., and M. Groudine. 1986. Transcriptional regulation of the human cytomegalovirus major immediate-early gene is associated with induction of DNaseI hypersensitive sites. Mol. Cell. Biol. 6:452-461.

15. Nelson, J. A., C. Reynolds-Kohler, and B. Smith. 1987. Negative and positive regulation by a short segment in the 5 '-flanking region of the human cytomegalovirus major immediate-early gene. Mol. Cell. Biol. 7:4125-4129.

16. Rice, G. P. A., R. D. Schrier, and M. B. A. Oldstone. 1984. Cytomegalovirus infects human lymphocytes and monocytes: virus expression is limited to immediate-early gene products. Proc. Natl. Acad. Sci. USA 81:6134-6138.

17. Schrier, R. D., J. A. Nelson, and M. B. A. Oldstone. 1985. Detection of human cytomegalovirus in peripheral blood lymphocytes in natural infection. Science 320:1048-1051.

18. Siekevitz, M., S. F. Josephs, M. Dukovich, N. Peffer, F. WongStaal, and W. C. Greene. 1987. Activation of the HIV-1 LTR by cell mitogens and the trans-activator protein of HTLV-1. Science 238:1575-1578.

19. Stinski, M. F., and T. J. Roehr. 1985. Activation of the major immediate-early gene of human cytomegalovirus by cis-acting elements in the promoter-regulatory sequence and by virusspecific trans-acting components. J. Virol. 55:431-441.

20. Stinski, M. F., D. R. Thomson, R. M. Stenberg, and L. C. Goldstein. 1983. Organization and expression of the immediateearly genes of human cytomegalovirus. J. Virol. 46:1-14.

21. Thomson, D. R., R. M. Stenberg, W. F. Goins, and M. F. Stinski. 1984. Promoter-regulatory region of the major immediate-early gene of the human cytomegalovirus. Proc. Natl. Acad. Sci. USA 81:659-663. 